\title{
Start and trigger detector to of the ALICE experiment
}

\section{Alla Maevskaya for the ALICE collaboration}

Institute for Nuclear Research Russian Academy of Science

E-mail: allalinr.ru

ALICE T0 detector serves as a start, trigger, and luminosity detector for the ALICE experiment. The accurate interaction time is used as the precision start signal for the TOF detector. For pp interactions, T0 achieves a time resolution of $40 \mathrm{ps,}$, while for $\mathrm{Pb}-\mathrm{Pb}$ collisions, the resolution is $20 \mathrm{ps}$. T0 supplies five different trigger signals to the Central Trigger Processor. The most important of these is the T0 vertex, which gives, promptly and accurately, the position of the primary interaction vertex along the beam axis. The detector is also used for online luminosity monitoring with a fast feedback to the accelerator team. The detector has shown very good performance and stable operations at high interaction rates of up $400 \mathrm{kHz}$ during the first 3 years of exploitation. 


\section{Introduction}

One of the most important parts of the ALICE is the T0 detector, detector, a key component of the trigger system and which provides the collision time zero for time of flight measurements. The T0 detector provides up to five different trigger signals for physics selections, based on the online determination of the centrality and of vertex position of the collision, and for background rejection. During the first years of the LHC running, the detector has shown excellent performance and stable operations, both in $\mathrm{pp}$ and $\mathrm{Pb}-\mathrm{Pb}$ collisions and it has fully confirmed its central role for the ALICE data taking. Excellent time resolution was obtained both in $\mathrm{pp}(\sim 40 \mathrm{ps})$ and $\mathrm{Pb}-\mathrm{Pb}(\sim 20 \mathrm{ps})$.

\section{The ALICE detector}

The theory of strong interactions, Quantum Chromo Dynamics (QCD), predicts a phase transition at high temperature between hadronic matter, where quarks and gluons are confined inside hadrons, and a deconfined state of matter, the Quark-Gluon Plasma (QGP). The LHC experiment designed to study $\mathrm{Pb}-\mathrm{Pb}$ collisions is the ALICE experiment. Its main difference with respect to the other LHC experiment is its excellent Particle IDentification (PID) capability. Thanks to it, ALICE is able to investigate the formation and the properties of the QGP through a large variety of signatures [1].

The ALICE detectors can be classified in three groups:

- Barrel detectors: they are placed in a solenoid which provides the experiment with a $0.5 \mathrm{~T}$ magnetic field and covers the pseudorapidity interval $-0.9 \leq \eta \leq 0.9$. They are dedicated to the vertex reconstruction, tracking, particle identification and momentum measurement. Going radially outward from the interaction region, ALICE consists of the following detectors:

- Inner Tracking System (ITS);

- Time Projection Chamber (TPC);

- Transition Radiation Detector (TRD);

- Time of Flight (TOF).

In the mid-rapidity region there are also two detectors with limited acceptance:

- High-Momentum Particle Identification Detector (HMPID);

- PHOton Spectrometer (PHOS);

In addition, the ElectroMagnetic CALorimeter (EMCAL) is present.

- Muon spectrometer, placed in the forward pseudorapidity region $(-4.0 \leq \eta \leq-2.5)$ consists of a dipole magnet and tracking and trigger chambers. It is optimized to reconstruct heavy quark resonances (such as $\mathrm{J} / \psi$ through their $\mu+\mu-$ decay channel) and single muons; 
- Forward detectors are placed in the high pseudorapidity region and are used for triggering or to measure global event characteristics, such as:

- Time Zero (T0) to measure the event time and provide Level-0 triggers when the vertex is within the preset value ;

- V0 to reject the beam-gas background and to trigger minimum bias events;

- Forward Multiplicity Detector (FMD) to provide multiplicity information over a large fraction of the solid angle $(-3.4 \leq \eta \leq-1.7$ and $1.7 \leq \leq 5))$

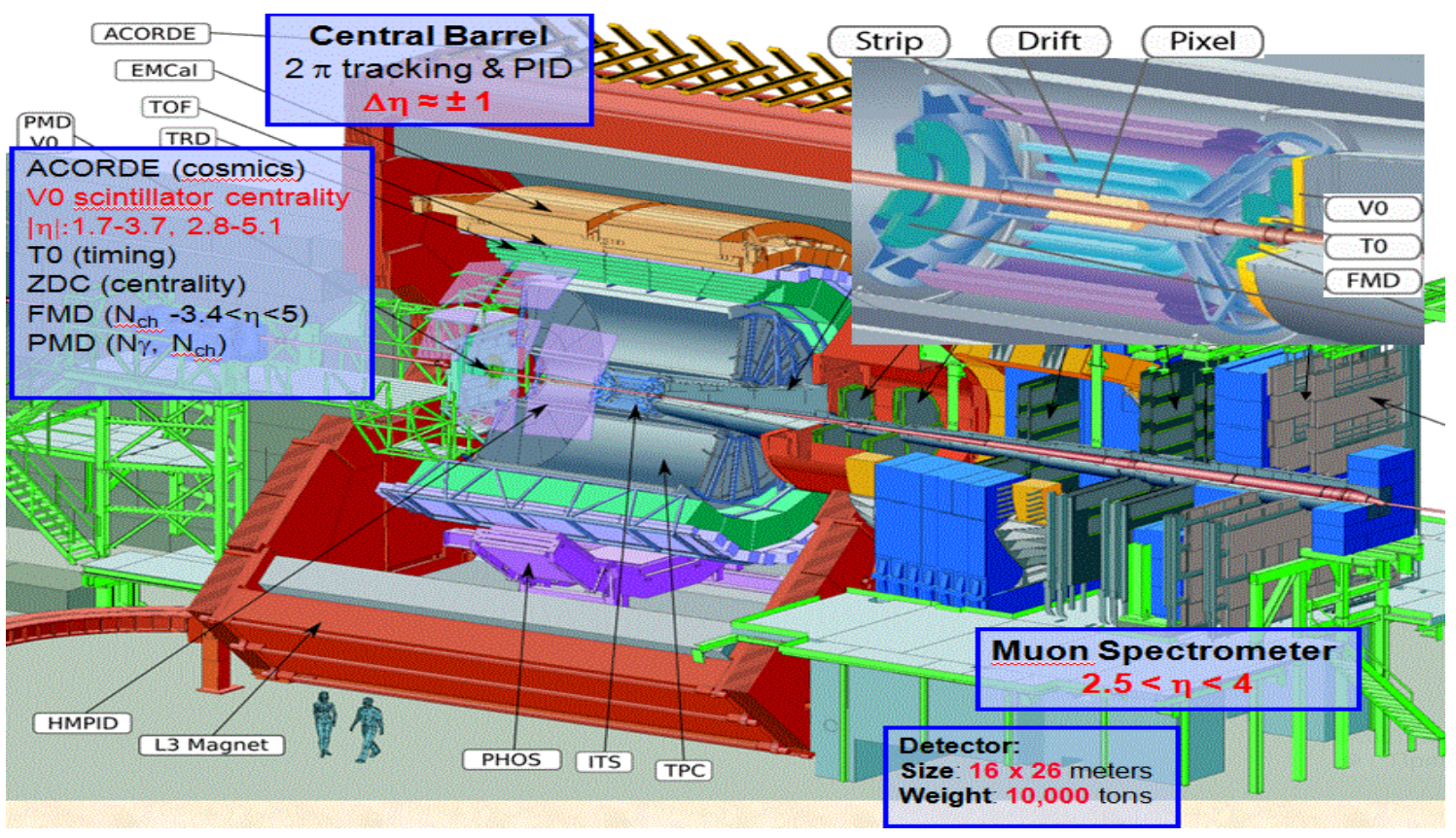

Figure 1. The ALICE detector overview.

\section{The T0 detector}

The T0 detector [2] was designed to generate a start-time (T0) signal for the Time-OfFlight (TOF) detector. This signal is independent of vertex position and corresponds to the real time of the collision. The T0 detector can also

- measure the vertex position (with $1 \mathrm{~cm}$ precision) and provide Level-0 triggers when the vertex is within the preset value;

- generate «wake-up» signal for TRD detector;

- measure luminosity and provide fast feedback to LHC.

The detector consists of two arrays of 12 Cherenkov counters. Each counter is based on fine-mesh photomultiplier tube coupled to a quartz radiator $20 \mathrm{~mm}$ in diameter and 20 $\mathrm{mm}$ thick. The two arrays are placed at $70 \mathrm{~cm}(\mathrm{~T} 0-\mathrm{C})$ and $374 \mathrm{~cm}(\mathrm{~T} 0-\mathrm{A})$ from the 
interaction point, respectively (Figure 1.25) and covered pseudorapidity region -3.28 $<\eta<-2.97$ with T0-C and $4.61<\eta<4.92$ with T0-A.
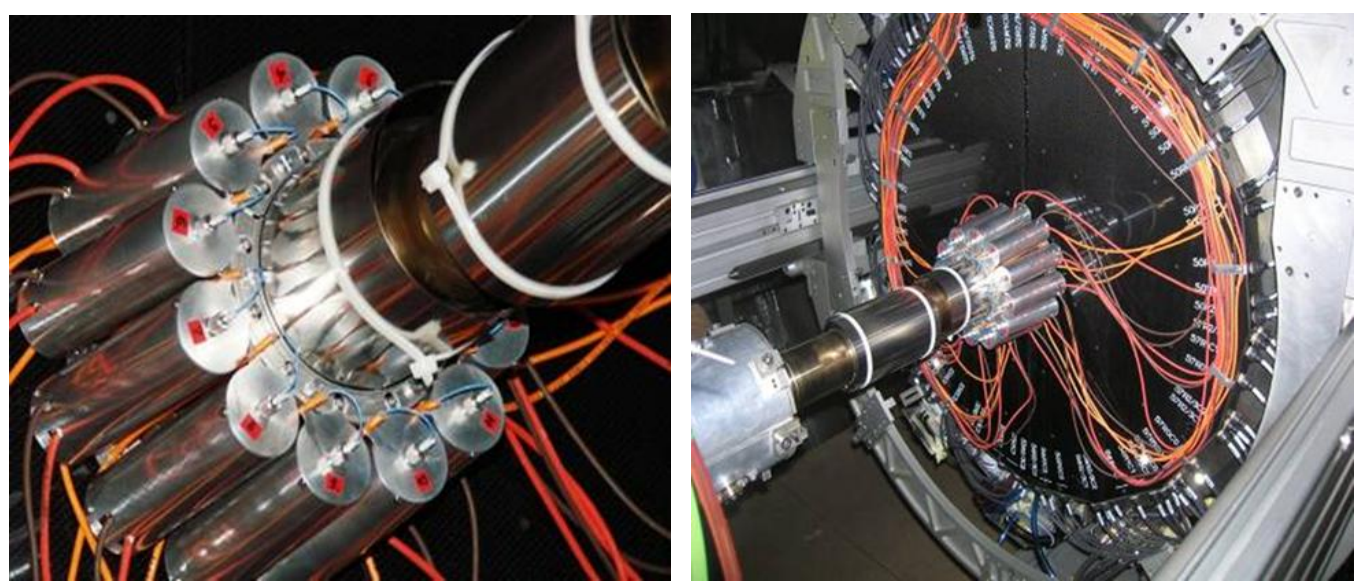

Figure 2. T0-C detector mounted around the beam pipe

\subsection{Start-time for TOF}

The main goal of T0 detector is to produce start-time signal independent on vertex position - $(\mathrm{T} 0 \mathrm{~A}+\mathrm{T} 0 \mathrm{C}) / 2$. For low multiplicity events, when only one counter produced a signal, T0A or T0C can be used after correcting for the z-position of the vertex measured by the ITS with an accuracy $\sim 50 \mu \mathrm{m}$. Start-time resolutions of (T0A+T0C)/2 are better than $40 \mathrm{ps}$ for $\mathrm{pp}$ and $20 \mathrm{ps}$ for $\mathrm{Pb}-\mathrm{Pb}$ collisions. Achieving such high resolutions requires good automatic run-by-run calibrations.

The T0 detector calibration procedure consists of online and offline parts.

The online run-by-run calibrations are based on raw data. Rough gain and slewing corrections are determined before the start of a run period. These are done using a set of laser runs, where the laser intensity is varied. The results are stored into Offline Calibration Database (OCDB).

ALICE calibration strategy requires 2 steps of offline calibration based on reconstructed ESD( Event Summary Data).

The mean time value for each PMT, after slewing correction, is optimized for the MB trigger and written to the OCDB for each channel. Subtracting the mean time value from each measured time signal during reconstruction places all time signals around zero and gives the fastest particle for each event. These time signals corrected with ITS vertex are T0A and T0C. (T0A+T0C)/2, T0A and T0C positions are written in 
OCDB. During reconstruction, T0A, T0C, and (T0A+T0C)/2 are shifted by the value form the OCDB, setting the start time to zero.

Figure 3, left, shows T0A, T0C and (T0A+T0C)/2 ready to be used by TOF detector as start-time. The right plot shows the distribution (T0A-T0C)/2 corrected by the ITS vertex position. Sigma of this distribution is the resolution of (T0A+T0C)/2 signal.
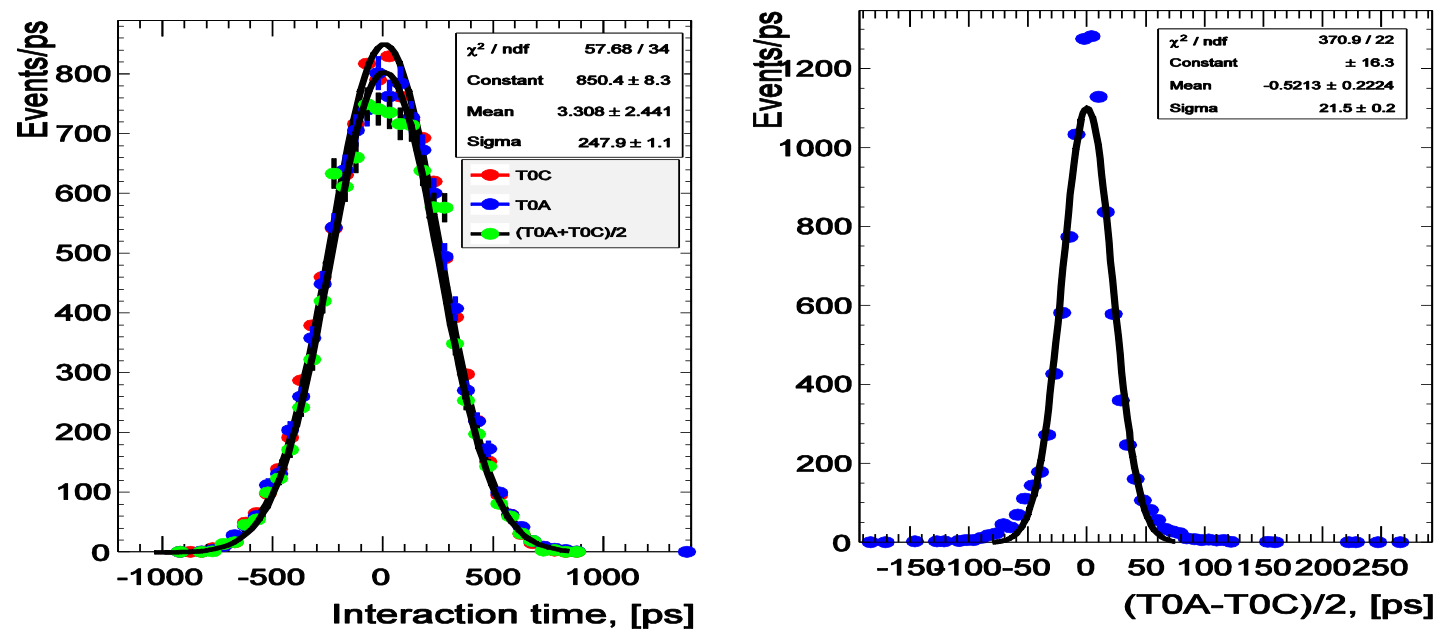

Figure 3. Left :T0A, T0C and (T0A+T0C)/2 distribution for $\mathrm{Pb}-\mathrm{Pb}$ run 170315. Right: $(\mathrm{T} 0 \mathrm{~A}+\mathrm{T} 0 \mathrm{C}) / 2$ resolution

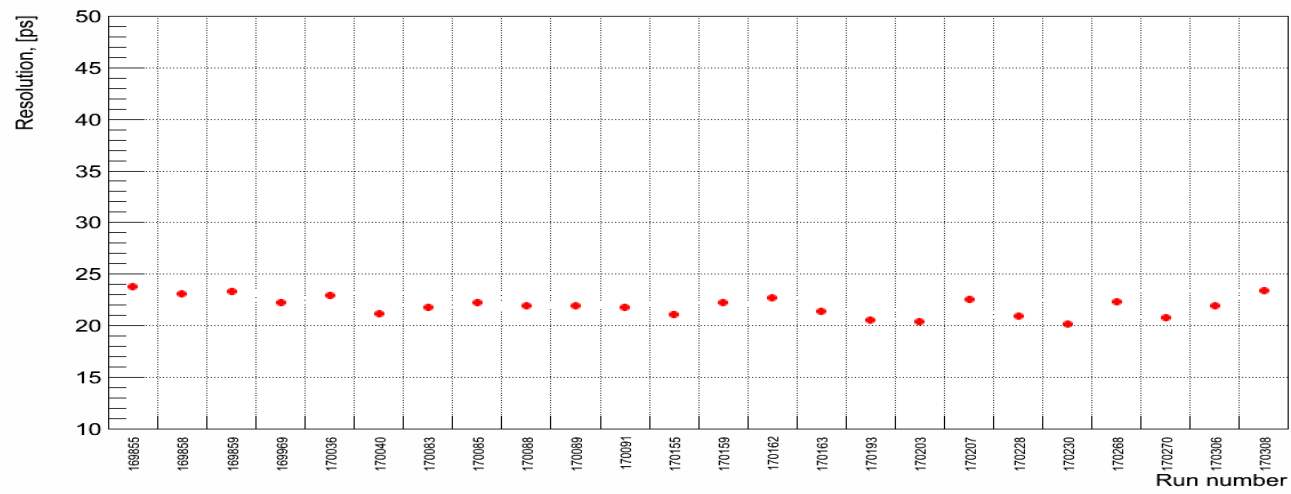

Figure 4. Trending histogram of (T0A+T0C)/2 vs run number for $\mathrm{Pb}-\mathrm{Pb} 2011$ period 
Figure 4 shows the stability of the T0 resolution for $\mathrm{Pb}-\mathrm{Pb} 2011$ runs. The detector resolution did not change over the data taking periods; no aging effects have been observed.

T0 efficiency depends on event multiplicity. For $\mathrm{Pb}-\mathrm{Pb}$ collisions it is around $100 \%$ for events with centrality below $70 \%$ and drops to $65 \%$ for T0A and T0C and $35 \%$ (T0A+T0C)/2 for events with centrality $90 \%$. For $7 \mathrm{TeV}$ proton collisions T0A or T0C signals were provided with efficiency $\sim 70 \%$ and (T0A+T0C)/2 with efficiency $50 \%$.

\subsection{T0 trigger signals}

The trigger functions requested from $\mathrm{T} 0$ are as follows:

- to measure the approximate vertex position;

- to give a rough estimate of event multiplicity;

- to inform that at least one of the arms of the T0 detector has registered a valid pulse.

The T0 vertex trigger (OTVX) uses a timing cut on the T0A-T0C difference to reject background and satellite collisions. It is used as an interaction trigger for running with high interaction rates $-400 \mathrm{kHz}$ in 2012 proton runs. In $\mathrm{Pb}-\mathrm{Pb}$ runs in 2011, it was also used for online selection of central and semi-central event. In addition for offline selection flags are written:

- T0 pile-up flag when more than one interactions were observed in interval -70ns +1130 ns from triggering event

- T0 background flag if we have both signal from T0-A and T0-C, but with vertex was out of given interval (OTVX signal -off)

Figure 5 shows T0 vertex trigger efficiency during $2011 \mathrm{~Pb}-\mathrm{Pb}$ runs for central $(0-10 \%)$ and semi-central events (10-50\%).

Figure 6 shows how well T0 vertex trigger rejects background and satellite collisions : left plot shows start-time (T0A+T0C)/2 vs T0 vertex distribution for all events, right plot - after T0 vertex trigger applied. 


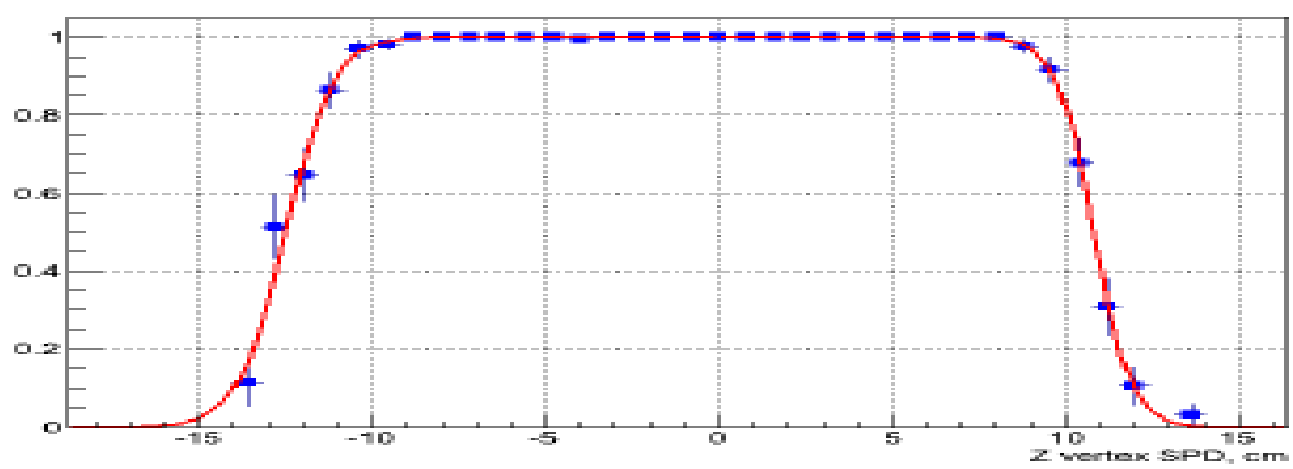

Figure 5. T0 vertex trigger efficiency during $2011 \mathrm{~Pb}-\mathrm{Pb}$ runs for central and semicentral events(0-50\%).
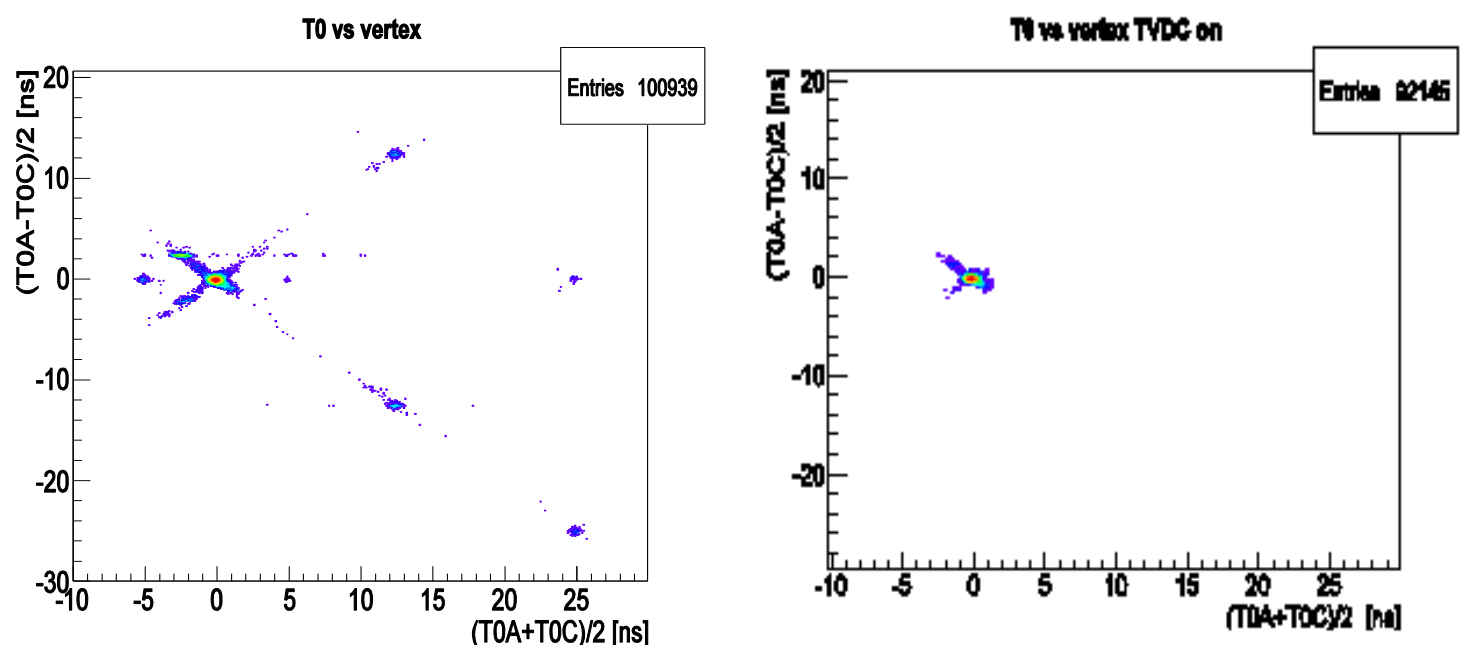

Figure 6. Example of background rejection with the T0 vertex trigger. Left plot: all events, right plot: with $\mathrm{T} 0$ vertex trigger $\mathrm{ON}$.

\subsection{Luminosity monitoring}

The T0 detector is used for online monitoring of the luminosity. Figure 7 shows a snapshot of the the ALICE LHC interface during the 2012 pp run. Luminosity is estimated from the measured rates. These are displayed and monitored ONLINE and then archived. This information is sent to the LHC for beam tuning, optimizing beam conditions and establishing proper run conditions. 


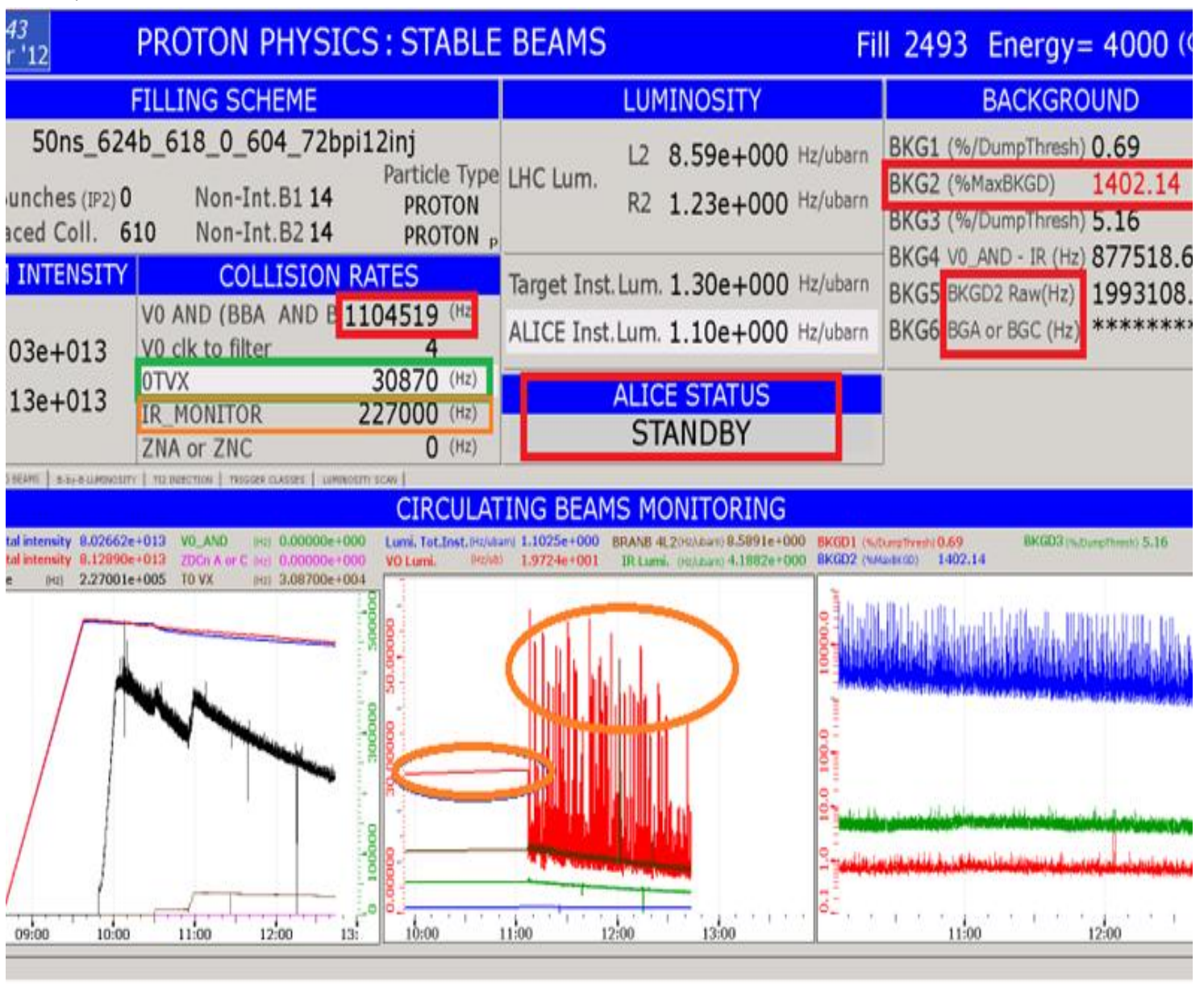

Figure 7. ALICE LHC interface snapshot.

\section{Conclusion}

The T0 detector, based on quartz Cherenkov technology has shown excellent performance and stable operations both in $\mathrm{pp}$ and $\mathrm{Pb}-\mathrm{Pb}$ collisions.

The work was supported by the Russian Academy of Sciences and by the Russian Foundation for Basic Research ( grant № 12-02-91508-CERN-a ).

\section{References}

[1] K. Aamodt et al., (ALICE Collaboration), JINST 3, (2008) S08002

[2] K. Aamodt et al. (ALICE), JINST 3, S08002 (2008) 